\title{
On Budget-Balanced Group-Strategyproof Cost-Sharing Mechanisms
}

\author{
Nicole Immorlica
}

\author{
Emmanouil Pountourakis
}

\begin{abstract}
A cost-sharing mechanism defines how to share the cost of a service among serviced customers. It solicits bids from potential customers and selects a subset of customers to serve and a price to charge each of them. The mechanism is group-strategyproof if no subset of customers can gain by lying about their values. There is a rich literature that designs group-strategyproof cost-sharing mechanisms using schemes that satisfy a property called cross-monotonicity. Unfortunately, Immorlica et al showed that for many services, cross-monotonic schemes are provably not budget-balanced, i.e., they can recover only a fraction of the cost. While cross-monotonicity is a sufficient condition for designing group-strategyproof mechanisms, it is not necessary. Pountourakis and Vidali recently provided a complete characterization of group-strategyproof mechanisms. Using their characterization, we construct a fully budget-balanced group-strategyproof mechanism for the edge-cover problem. This improves upon the cross-monotonic approach which can recover only half the cost, and provides a proof-of-concept as to the usefullness of the complete characterization. This raises the question of whether all "natural" problems have budget-balanced group-strategyproof mechanisms. We answer this question in the negative by designing a set-cover instance in which no group-strategyproof mechanism can recover more than a (18/19)-fraction of the cost.
\end{abstract}




\section{Introduction}

In cost-sharing problems, a service provider faces a set of potential customers, each of which has a private value for the service. The provider must select a subset of customers to serve, and a price to charge each of them. To this end, he defines a mechanism that solicits bids from potential customers and, based on these bids, outputs the serviced subset and corresponding prices. To cover the cost of providing service, he looks for a mechanism that is budget-balanced, that is the sum of prices equals the cost of service for every input bid vector.

A central goal in mechanism design is to define mechanisms that are strategyproof in that no agent can gain by misreporting his value. This guarantees that the equilibrium bidding strategy of agents is robust and so the mechanism behaves as predicted. In cost-sharing problems, there is an inherent cooperative aspect: the cost of service changes drastically depending on which subset is serviced and so groups of agents may have aligned interests. In these problems, it makes sense to ask for an even more robust solution concept, group-strategyproofness. In a group-strategyproof mechanism, no group of agents can mutually gain by misreporting their values.

Group-strategyproofness is a very strong requirement. Nonetheless, there is a rich literature defining group-strategyproof mechanisms for various cost-sharing problems [4, 7, 6, 11]. All these papers use the same general technique. They define a cost-sharing scheme which, given any subset of customers, defines the price each of them would have to pay if that subset was serviced. They then turn this scheme into a mechanism by applying a procedure of Moulin [9]. The resulting mechanism is group-strategyproof so long as the underlying cost-sharing scheme satisfies a property called cross-monotonicity. Intuitively, cross-monotonicity requires that as more agents are serviced, the price to each decreases. If the cross-monotonic cost-sharing scheme is (approximately) budgetbalanced on every subset of customers, then the resulting group-strategyproof mechanism is also (approximately) budget-balanced.

Unfortunately, the use of this technique comes at a cost. While submodular cost functions always have fully budget-balanced cross-monotonic cost-sharing schemes [5], and many combinatorial optimization problems have approximately budget-balanced schemes $[4,7,6,11]$, Immorlica et al. [5] prove that cross-monotonicity fundamentally limits achievable budget-balance factors for many combinatorial optimization problems. They also note that, while cross-monotonicity is a sufficient condition for giving rise to group-strategyproof mechanisms, it is not necessary. This left open the question of whether another approach might enable the design of group-strategyproof mechanisms with better budget-balance factors.

In a recent work, Pountourakis and Vidali [13] gave a complete characterization of groupstrategyproof mechanisms. Their characterization is based on cost-sharing schemes that satisfy three technical properties. They then give a procedure that converts any such (approximately) budget-balanced cost-sharing scheme into an (approximately) budget-balanced group-strategyproof mechanism. ${ }^{1}$

In this work, we use the edge-cover cost-sharing problem to showcase the Pountourakis-Vidali technique of designing group-strategyproof mechanisms. In the edge-cover problem, the agents are the vertices of a graph, and the cost of a subset is the minimum number of edges that must be selected in order to cover every agent in the subset. The problem models, for example, assigning people to rooms either as a single occupant or with a compatible roommate (as defined by the edges of the graph).

\footnotetext{
${ }^{1}$ In general, this procedure is not known to be polynomial-time.
} 
The results of Immorlica et al. [5] show that the best budget-balance factor of any crossmonotonic cost-sharing scheme for edge-cover is just $1 / 2$. Using the characterization of Pountourakis and Vidali [13], we design a fully budget-balanced group-strategyproof mechanism for edge-cover. Thus our result improves upon any group-strategyproof mechanism designed using the standard cross-monotonic technique, thereby demonstrating the significance of the full characterization. Furthermore, the cost-sharing scheme that we define is very intuitive: for a given subset of agents, we compute a lexicographically first maximum matching of that subset, charge each matched agent a price of $1 / 2$, and charge each remaining agent in the subset a price of 1 . This natural scheme is not cross-monotonic. However, using a key lemma regarding alternating paths of certain matchings, we are able to prove that this scheme does satisfy the characterization of Pountourakis and Vidali [13].

We also show that not all problems have fully budget-balanced group-strategyproof mechanisms. This is fairly obvious when one allows arbitrary (e.g., non-monotone) cost functions. ${ }^{2}$ In this paper, we prove this result for the natural monotone cost function defined by the set cover problem, a generalization of the edge cover problem. For set cover, Immorlica et al [5] showed a bound of $n^{-1 / 2}$ (where $n$ is the number of agents) on the budget-balance factor of cross-monotonic cost-sharing schemes, implying that the standard technique for designing group-strategyproof mechanisms is highly impractical. This negative result is particularly disturbing in light of the fact that there exists a trivial fully budget balanced strategyproof mechanism (see Example 4.1 [5]) for any nondecreasing cost-function if we dont take computational limits into consideration. Even imposing computational limits, Devanur et al [3] obtain a $O(1 / \log n)$-budget balanced mechanism that is strategyproof but not group-strategyproof. In our work we are interested in bounding the power of group-strategyproofness without any computational assumption. We present a set-cover instance, where there is no cost-sharing scheme satisfying the characterization of Pountourakis and Vidali [13] with budget-balance factor better than 18/19. Since this characterization does not take computational constraint into consideration this implies a bound for every group-strategyproof mechanism independent of its running time.

Our proof uses an interesting interesting property of the cost-sharing schemes of Pountourakis and Vidali [13]. Namely, we define agents to be good, bad, or neutral for one another at particular sets depending on how their costs change when each is removed. ${ }^{3}$ If the cost to Jack increases when Jill is removed from the current set of serviced agents, then we say Jill is good for Jack at that set. On the other hand, if the cost to Jack decreases when Jill is removed, then Jill is bad for Jack at that set. If neither happens (i.e., the cost to Jack remains the same), then Jill is neutral. We derive natural conditions on the good/bad/neutral relations of agents which we believe to be of independent interest for future work in proving lower-bounds on group-strategyproof mechanisms.

Finally, we would like to note that while we try to deal with the limitations of cross-monotonic mechanisms by exploiting the full power of group-strategyprofness, another approach that has been followed so far was to relax group-strategyproofness. In particular, Mehta et al. [8] defined the notion of weak group-strategyproonfess and proposed a general framework to design mechanisms that satisfy this property. This framework has been used to design mechanism with better budget balance guarantees for many combinatorial problems. [8, 1]. Specifically, cost-sharing schemes used by Mehta et al. [8] were naturally derived by primal-dual schemes without any refinement

\footnotetext{
${ }^{2}$ See, for example, Pountourakis and Vidali [13].

${ }^{3}$ This corresponds to the notion of positive, negative, and neutral elements of Immorlica et al. [5], and one of the properties we derive is precisely the property of semi-cross-monotonicity as derived in that paper.
} 
that would ensure cross-monotonicity [11]. However, as we argue in Section 5 these cost-sharing schemes fail to satisfy the necessary condition to give rise to group-strategyproofness; hence, ideas from this literature cannot be directly applied to group-strategyproof mechanism design.

Related Work. In addition to the literature on cost-sharing mechanisms mentioned above, our work is related to the literature on combinatorial public projects. This problem was introduced by Papadimitriou et al. [12] who assume that a set of agents is interested in sharing a number of resources. Each agent has a private valuation for each subset of these resources. For some given $k$, a mechanism has to choose based on the valuations of the agent a set of $k$ resources so as to maximize the social welfare.

Papadimitriou et al. [12] provide communication and computational bounds for every strategyproof mechanism that solves this problem when the valuation functions satisfy submodularity. Schapira and Singer [14] also provide similar bounds without the constraint of truthfulness, but slightly relaxing submodularity of valuation functions. Finally, Buchfuher et al. [2] study similar questions for sub-additive valuations and provide various upper and lower bound for specific valuation function classes.

This problem differs from cost-sharing in the sense that there are multiple resources the mechanism is called to choose upon, however, all the agents are going to share them. Moreover, they are interested in maximizing social welfare rather than budget balance. However, both of these problems have applications to resource sharing and particularly network formation.

\section{Model}

A set of agents $\mathcal{A}=\{1,2, \ldots, n\}$ is interested in receiving a service. Each agent $i$ has a private type $v_{i}$, which is her valuation for receiving the service. A cost-sharing mechanism inputs a bid $b_{i}$ for each agent $i$ and outputs the subset of agents $Q \subseteq \mathcal{A}$ that receive service and the price $p_{i}$ that each agent $i$ pays. Assuming quasi-linear utilities, each agent wants to maximize the quantity $v_{i} x_{i}-p_{i}$ where $x_{i}=1$ if $i \in Q$ and $x_{i}=0$ if $i \notin Q$. We concentrate on mechanisms that satisfy the following conditions $[9,10]$ :

- Voluntary Participation (VP): An agent that is not serviced is not charged $\left(i \notin Q \Rightarrow p_{i}=0\right)$ and a serviced agent is never charged more than her bid $\left(i \in Q \Rightarrow p_{i} \leq b_{i}\right)$.

- No Positive Transfer (NPT): The payment of each agent $i$ is non-negative $\left(p_{i} \geq 0\right.$ for all $i$ ).

- Consumer Sovereignty (CS): For each agent $i$ there exists a value $b_{i}^{*} \in \mathbb{R}$ such that if she bids $b_{i}^{*}$, then it is guaranteed that agent $i$ will receive the service no matter what the other agents bid.

We also assume that the agents can bid in a way that they will definitely not receive the service. This can be done by allowing negative bids. Then VP implies that an agent that reports a negative amount has to be charged a negative amount if she is serviced, which is prohibited by NPT.

We are interested in mechanisms that are group-strategyproof (GSP). A mechanism is GSP if for every two valuation vectors $v, v^{\prime}$ and every coalition of agents $S \subseteq \mathcal{A}$, satisfying $v_{i}=v_{i}^{\prime}$ for all $i \notin S$, one of the following is true: (a) There is some $i \in S$, such that $v_{i} x_{i}^{\prime}-p_{i}<v_{i} x_{i}-p_{i}$ or (b) for all $i \in S$, it holds that $v_{i} x_{i}^{\prime}-p_{i}=v_{i} x_{i}-p_{i}$.

We also assume the existence of an underlyng cost-function $C: 2^{\mathcal{A}} \rightarrow \mathbb{R}^{+} \cup\{0\}$, where $C(S)$ specifies the cost of providing service to all agents in $S$. We say that a mechanism is $\alpha$-budget 
balanced with respect to $C$ if for all $b, \alpha C(Q) \leq \sum_{i \in \mathcal{A}} p_{i} \leq C(Q)$, where $Q$ and $\left\{p_{i}\right\}$ are the prices and allocation output by the mechanism on input $b$.

\subsection{Characterization}

A cost-sharing scheme $\xi: \mathcal{A} \times 2^{\mathcal{A}} \rightarrow \mathbb{R}$ is a function that takes as input an agent and a set and outputs a real number. The amount $\xi(i, S)$ can be viewed as the payment of agent $i$ when the set of agents $S$ receives the service. ${ }^{4}$ A cost-sharing scheme is $\alpha$-budget balanced with respect to $C$ if for all $S, \alpha C(S) \leq \sum_{i \in S} \xi(i, S) \leq C(S)$.

A property of the cost-sharing scheme, namely cross-monotonicity, has played a central role in the literature. Intuitively, cross-monotonicity requires that the cost-share of an agent can not increase as the serviced set grows. Moulin $[9,10]$ showed that given a cross-monotone cost-sharing scheme we can construct a group-strategyproof mechanism with a budget-balance factor equal to that of the cost-sharing scheme. Moreover, if the underlying cost function is submodular then there exist a perfectly budget balanced cost-sharing scheme. However, when the cost function is given by the cost of the optimal solution of an optimization problem, the cost function is often not submodular. Immorlica et al. [5] proved bounds on the budget balance factor of cross-monotonic cost-sharing schemes. This gave rise to the question of whether there are group-strategyproof mechanisms for such problems with better budget-balance properties. As a step towards answering this question, Pountourakis and Vidali [13] provided a complete characterization of the cost-sharing schemes that can give rise to group-strategyproof mechanisms. Let $\xi^{*}(i, L, U)$ be the minimum payment of player $i$ for getting serviced when the serviced set is between sets $L$ and $U$, i.e., $\xi^{*}(i, L, U):=\min _{\{L \subseteq S \subseteq U, i \in S\}} \xi(i, S)$.

Theorem 1 (Pountourakis and Vidali [13]). A cost-sharing scheme $\xi$ can give rise to a groupstrategyproof mechanism if and only if for every $L \subseteq U \subseteq \mathcal{A}$ it satisfies the following three properties.

(a) There exists a set $S$ with $L \subseteq S \subseteq U$, such that for all $i \in S$, we have $\xi(i, S)=\xi^{*}(i, L, U)$.

(b) For each player $i \in U \backslash L$ there exists one set $S_{i}$ with $i \in S_{i}$ and $L \subseteq S_{i} \subseteq U$, such that for all $j \in S_{i} \backslash L$, we have $\xi\left(j, S_{i}\right)=\xi^{*}(j, L, U)$.

(Since $i \in S_{i} \backslash L$, it holds that $\xi\left(i, S_{i}\right)=\xi^{*}(i, L, U)$.)

(c) If for some $C \subset U$ there is a player $j \in C$ with $\xi(j, C)<\xi^{*}(j, L, U)$ (obviously $L \nsubseteq C$ ), then there exists a set $T \neq \emptyset$ with $T \subseteq L \backslash C$, such that for all $i \in T, \xi(i, C \cup T)=\xi^{*}(i, L, U)$.

To make our arguments and the use of this characterization more intuitive we introduce some additional definitions and properties that are implied by this theorem and are themselves of independent interest. Given a set $S$ and agents $i, j \in S$ we say that agent $i$ is good for $j$ at $S$ if $\xi(i, S)<\xi(i, S \backslash\{j\})$, agent $i$ is bad for $j$ at $S$ if $\xi(i, S)>\xi(i, S \backslash\{j\})$, and agent $i$ is neutral to agent $j$ at $S$ otherwise. The characterization implies the following.

Proposition 1. Given a cost-sharing scheme $\xi$ that can give rise to a group-strategyproof mechanism, the following hold for every $S$.

\footnotetext{
${ }^{4}$ This is a restrictive form of a payment policy as we exclude the possibility of charging different values given two different bid vectors where the mechanism provides the service to the same set of agents. Nevertheless, this is without loss of generality for the mechanisms of our setting [5].
} 
(a) Given $i \in S$ then either $i$ is bad or neutral for every $j \in S$ at $S$, or $i$ is good or neutral for every $j \in S$ at $S .^{5}$

(b) Given $i, j \in S$, if $i$ is bad for $j$ at $S$ then $j$ is neutral for $i$ at $S$.

(c) Given $i, j, k \in S$, if $i$ is bad for $j$ at $S$ and $j$ is bad for $k$ at $S$, then $i$ is bad for $k$ at $S$.

Proof.

(a): The statement is equivalent to requiring that $i$ is not both good for some $j \in S$ at $S$ and bad to another $k \in S$ at $S$. Assume for the purpose of contradiction that this holds. By property (a) of Theorem 1 for $L=S \backslash\{i\}$ and $U=S$ either at $S \backslash\{i\}$ or at $S$ every agent should be charged the minimum value among these two sets. However, this cannot true since from our assumption $\xi(j, S)<\xi(j, S \backslash\{i\})$ and $\xi(k, S \backslash\{i\})<\xi(k, S){ }^{6}$

(b): First assume that $i$ is bad for $j$ at $S$ and $j$ is bad for $i$ at $S$. We derive a contradiction with property (c) of Theorem 1. To that end, let $L=S \backslash\{j\}, U=S$, and $C=S \backslash\{i\}$. Note that, since $i$ is bad for $j, \xi(j, C)=\xi(j, S \backslash\{i\})<\xi(j, S)=\xi^{*}(j, L, U)$. Thus the conditions of property (c) apply and require that there is a nonempty $T \subseteq L \backslash C$ such that for all $k \in T, \xi(k, C \cup T)=\xi^{*}(k, L, U)$. For our setting of $L$ and $C$, the only choice for $T$ is $\{i\}$. But for $T=\{i\}, \xi(i, C \cup T)=\xi(i, S)>$ $\xi(i, S \backslash\{j\})=\xi^{*}(i, L, U)$ as $j$ is bad for $i$ at $S$. Thus, there is no choice for $T$ satisfying the property.

Next assume that $i$ is bad for $j$ at $S$ and $j$ is good for $i$ at $S$. We derive a contradiction with property (b) of Theorem 1. Let $L=S \backslash\{i, j\}$ and $U=S$. By the property, there must be a set $S_{i}$ where $i \in S_{i}, L \subseteq S_{i} \subseteq U$, and all $k \in S_{i} \backslash L$ have cost-share $\xi\left(k, S_{i}\right)=\xi^{*}(k, L, U)$. Now since $j$ is good for $i, \xi(i, S)<\xi(i, S \backslash\{j\})$ and so $\xi(i, S)=\xi^{*}(j, L, U)$. Hence the only choice for $S_{i}$ is $S$. However, agent $j$ is not happy in this set: $\xi\left(j, S_{i}\right)=\xi(j, S)>\xi(j, S \backslash\{i\})=\xi^{*}(j, L, U)$ since $i$ is bad for $j$. Thus, there is no choice for $S_{i}$ satisfying the property.

(c): Assume that the converse holds, i.e., agent $i$ is bad for $j$ at $S$, agent $j$ is bad for $k$ at $S$, and yet agent $i$ is not bad for agent $k$ at $S$. We derive a contradiction with property (c) of Theorem 1 . Let $L=S \backslash\{i\}, U=S$, and $C=S \backslash\{j\}$. Note that, since $i$ is not bad for $k, \xi(k, S \backslash\{i\}) \geq \xi(k, S)$,

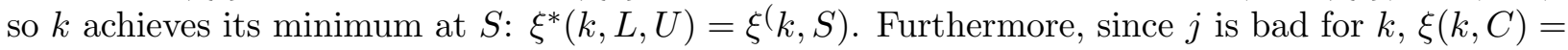
$\xi(k, S \backslash\{j\})<\xi(k, S)=\xi^{*}(k, L, U)$. Thus the conditions of property (c) apply and require that there is a nonempty $T \subseteq L \backslash C$ such that for all $l \in T, \xi(l, C \cup T)=\xi^{*}(l, L, U)$. For our setting of $L$ and $C$, the only choice for $T$ is $\{j\}$. But for $T=\{j\}, \xi(j, C \cup T)=\xi(j, S)>\xi(j, S \backslash\{i\})=\xi^{*}(j, L, U)$ as $i$ is bad for $j$ at $S$. Thus, there is no choice for $T$ satisfying the property.

\subsection{Allocation}

Pountourakis and Vidali [13] provided a characterization of the allocation functions that can be coupled with a cost-sharing scheme satisfying the properties of Theorem 1 to yield a groupstrategyproof mechanism.

Theorem 2 (Pountourakis and Vidali [13]). If $\xi$ is a cost-sharing scheme that satisfies the properties of Theorem 1, then for every bid vector $b$ there exists unique sets $L(b) \subseteq U(b) \subseteq \mathcal{A}$ such that

1. for all $i \in L(b), b_{i}>\xi^{*}(i, L(b), U(b))$,

\footnotetext{
${ }^{5}$ This is exactly the necessary property of semi-cross-monotonicity defined in Immorlica et al. [5].

${ }^{6}$ As indicated by this proof, semi-cross-monotonicity coincides with property (a) of Theorem 1 when restricted to sets where $|U \backslash L|=1$.
} 
2. for all $i \in U(b) \backslash L(b), b_{i}=\xi^{*}(i, L(b), U(b))$,

3. and for all $R \subseteq \mathcal{A} \backslash U(b)$, there exist $i \in R$ with $b_{i}<\xi^{*}(i, L(b), U(b) \cup R)$.

Furthermore, the mechanism that on input $b$ outputs allocation $Q=S$ and prices $p_{i}=\xi(i, S)$ where

1. $L(b) \subseteq S \subseteq U(b)$, and

2. for all $i \in S, \xi(i, S)=\xi^{*}(i, L(b), U(b))$ (such a set must exist by Theorem 1(a)),

is a group-strategyproof mechanism.

A way to implement the allocation is to exhaustively search for these sets $L(b)$ and $U(b)$ and a set $S$ that satisfy the properties of the theorem. It is still not known if there algorithm that implements this procedure with asymptotically better running time in general. However, in Section 3, we provide a fully budget-balanced cost-sharing scheme and corresponding polynomial-time allocation when the cost function is given by the edge-cover problem.

\section{Edge Cover}

In this section, we give a fully budget-balanced group-strategyproof mechanism for the edge-cover cost-sharing game. To do so, we derive a cost-sharing scheme that satisfies the conditions presented in Theorem 1. Previous work [5] implies that group-strategyproof mechanisms for edge-cover designed via cross-monotonic cost-sharing schemes are at best $1 / 2$-budget balanced. Thus, our work improves upon the previous results and demonstrates that the assumption of cross-monotonicity is not without loss for group-strategyproof mechanism design. We start with a definition of the edge cover game.

Definition 1. In the edge cover game we are given a graph $G=(V, E)$ with no isolated vertices. The agents in the game are the vertices $V$ of the graph $G$. Given a subset of vertices $S \subseteq V$, an edge-cover of $S$ is a subset of edges $F \subseteq E$ such that for all vertices $v \in S$ there is some edge $e \in F$ such that $v \in e$. The cost of a set $S$ is the minimum cardinality edge-cover of $S$.

In the following subsections, we first present a cost-sharing scheme for the edge cover game that provably gives rise to a group-strategyproof mechanism. We then show how to use this scheme to define a computationally efficient group-strategyproof mechanism for our problem.

\subsection{Cost-sharing Scheme}

Our cost-sharing scheme is based on the following well-known polynomial time algorithm for finding the minimum edge cover $F$ of a set $S$. Let $F$ be the set of edges in the maximum matching on $S$, and then for each vertex $v \in S$ uncovered by $F$, add to $F$ an edge $e$ adjacent to $v$. Based on this algorithm, a natural cost-sharing scheme is to charge each agent $v \in S$ a price of $1 / 2$ if $v$ is in the matching found by the algorithm, and 1 otherwise. The problem that arises with this cost-sharing scheme is the existence of multiple maxima matchings. The following example shows that if the tie-breaking choices of the algorithm are inconsistent between different inputs $S$, then the scheme fails to give rise to group-strategyproof mechanisms.

Example 1. Consider a star graph with four vertices $\left\{v_{0}, v_{1}, v_{2}, v_{3}\right\}$ where $v_{0}$ is the center of the star, i.e., $E=\left\{\left(v_{0}, v_{1}\right),\left(v_{0}, v_{2}\right),\left(v_{0}, v_{3}\right)\right\}$. For the set $\left\{v_{0}, v_{1}, v_{2}, v_{3}\right\}$ the maxima matchings have 
size one and consist of one of the three edges. Suppose the algorithm on input $\left\{v_{0}, v_{1}, v_{2}, v_{3}\right\}$ selects edge $e=\left(v_{0}, v_{1}\right)$ to be in the matching. This means that in the cost-sharing scheme the vertices $v_{0}$ and $v_{1}$ are charged $1 / 2$ and vertices $v_{2}$ and $v_{3}$ are charged 1.

Now consider the set $\left\{v_{0}, v_{1}, v_{2}\right\}$. Assume that this time the algorithm on input $\left\{v_{0}, v_{1}, v_{2}\right\}$ selects edge $e=\left(v_{0}, v_{2}\right)$ to be in the matching. Then the cost-sharing scheme charges vertices $v_{0}$ and $v_{2}$ a price of $1 / 2$ and vertex $v_{1}$ a price of 1 .

As a result, $\xi\left(v_{2},\left\{v_{0}, v_{1}, v_{2}, v_{3}\right\}\right)=1>1 / 2=\xi\left(v_{2},\left\{v_{0}, v_{1}, v_{2}\right\}\right)$ and $\xi\left(v_{1},\left\{v_{0}, v_{1}, v_{2}, v_{3}\right\}\right)=$ $1 / 2<1=\xi\left(v_{1},\left\{v_{0}, v_{1}, v_{2}\right\}\right)$. Thus $v_{3}$ is bad for $v_{2}$ at $\left\{v_{0}, v_{1}, v_{2}, v_{3}\right\}$ and good for $v_{1}$ at the same set. This contradicts property (a) of Proposition 1.

Since our construction favors the agents that are matched by the algorithm, it is important to have some consistency on the choice of matching. We resolve this issue by choosing the lexicographically first maximum matching.

Definition 2. Given $G=(V, E)$ on $m$ edges, label edges $E$ from 1 to $m$ arbitrarily. For a subset of vertices $S \subseteq V$, let $M_{S}$ denote the lexicographically first maximum matching according to the labeling. Moreover let $V\left(M_{S}\right)=\left\{v \mid \exists e \in M_{S}\right.$ s.t. $\left.v \in e\right\}$, i.e., $V\left(M_{S}\right)$ contains the vertices that are matched in $M_{S}$.

Note that the lexicographically first maximum matching $M_{S}$ of any set of vertices $S$ can be found efficiently, for example by assigning a weight of $\left(1+2^{i}\right) / 2^{m}$ to the $i$ 'th edge and then computing the maximum weight matching. We are now ready to formally define the cost-sharing scheme $\xi$. This cost-sharing scheme extends one introduced by Immorlica et al. [5] for an edge-cover instance on just three vertices as an example of a group-strategyproof mechanism without a cross-monotone cost-sharing scheme.

Definition 3. Let $G=(V, E)$. For every $S \subseteq V$ and every $i \in V$ we define

$$
\xi(i, S)=\left\{\begin{array}{cc}
0 & i \notin S \\
1 / 2 & i \in V\left(M_{S}\right) \\
1 & i \in S \backslash V\left(M_{S}\right)
\end{array}\right.
$$

By construction, the cost-sharing scheme of Definition 3 is 1-budget balanced (and therefore, by the results of Immorlica et al. [5], it is not cross-monotone). We show that it additionally satisfies all the necessary and sufficient conditions of group-strategyproofness.

Theorem 3. The cost-sharing scheme $\xi$ of Definition 3 satisfies all the necessary and sufficient conditions to give rise to a GSP mechanism. Consequently there is a 1-budget balanced GSP mechanism for the edge-cover problem.

The proof uses the characterization presented in Theorem 1. The main challenge is to show that for any lower set $L$ and upper set $U$, there is some intermediate set $S^{*}, L \subseteq S^{*} \subseteq U$, in which every agent in $S^{*}$ achieves his minimum cost-share among all intermediate sets (i.e., property (a)). Since cost-shares are always either 1 or $1 / 2$, this amounts to finding a set $S^{*}$ in which each agent $i \in S^{*}$ either has cost-share $1 / 2$, or has cost-share 1 for every intermediate set $S, L \subseteq S \subseteq U$.

The proof idea is as follows. We start with an arbitrary intermediate set $S$ and work our way towards $S^{*}$. First, we prove in the following lemmas that for any set $S$, we can discard agents with cost-share equal to 1 without changing the solution for the other agents. Thus starting from an 
arbitrary intermediate set $S$, we can work our way towards $S^{*}$ by discarding all agents in $S \backslash L$ with cost-share equal to 1 . This leaves the question of whether agents in $S \cap L$ are receiving their minimum cost-share among the intermediate sets. Unfortunately, this is not necessarily the case: there may be some unhappy agent $i \in L \cap S$ with cost-share equal to 1 who has a cost-share equal to $1 / 2$ in some other intermediate set $S_{i}$. In this case, we use the lexicographically first maximum matchings $M_{S}$ and $M_{S_{i}}$ to construct an alternating path starting from agent $i$. We prove that this alternating path ends in a node $j$ that can either be added to or deleted from $S$ in order to decrease the number of unhappy agents in $L \cap S$ (interestingly, agent $i$ may still be unhappy after this fix, but at least one agent becomes happy). In this way, starting from an arbitrary intermediate set $S$, we can walk towards $S^{*}$.

Before we proceed with the proof we provide two useful lemmas that help us iteratively manipulate sets. The first lemma and its corollary imply that we can discard elements with cost-share 1 from some set without affecting the cost-shares of the other elements.

Lemma 1. Consider some $S \subseteq V$. If there is some $i \in S$ such that $\xi(i, S)=1$, then for all $j \in S \backslash\{i\}, \xi(j, S)=\xi(j, S \backslash\{i\})$.

Proof. Since $\xi(i, S)=1$, agent $i$ must be uncovered by the maximum matching $M_{S}$, i.e., $i \notin V\left(M_{S}\right)$. Therefore, removing agent $i$ does not decrease the cardinality of $M_{S}$, and so the set of maxima matchings of $S \backslash\{i\}$ is a subset of the maxima matchings of $S$. As a result, $M_{S}$ is still the lexicographically first matching, i.e., $M_{S \backslash\{i\}}=M_{S}$. Part (a) follows by definition of $\xi$.

The following corollary follows immediately by applying Lemma 1 inductively.

Corollary 1. Consider an $S \subseteq V$ and $a T \subseteq\{i \mid \xi(i, S)=1\}$. Then for all $i \in S \backslash T, \xi(i, S)=$ $\xi(i, S \backslash T)$.

The second lemma is the key lemma. It says that whenever there is an unhappy (i.e., unmatched) element $i$ at some current set $S$, then there is another element $j$ that can be added or deleted in order to increase the total number of happy (i.e., matched) elements (not counting $j$ ).

Lemma 2. Let $L \subseteq U \subseteq \mathcal{A}$ and $i \in U$. Consider any set $S$ with $L \subseteq S \subseteq U$ such that $\xi(i, S)>$ $\xi^{*}(i, L, U)$. Then either

- there is some $j \in S \backslash L$ such that the number of matched elements does not decrease when we remove $j$, i.e., $|V(S)| \leq|V(S \backslash\{j\})|$, and $j$ was matched in $M_{S}$ (hence the number of matched elements restricted to $S \backslash\{j\}$ strictly increases),

- or there is some $j \in U \backslash S$ such that the number of matched elements strictly increases when we add $j$, i.e., $|V(S)|<|V(S \cup\{j\})|$ (and since the number of matched elements is always even, the number of matched elements restricted to $S$ strictly increases as well).

Proof. Let $i$ and $S$ be as in the theorem statement, and let $S_{i}, L \subseteq S_{i} \subseteq U$, be a set where $i$ achieves its minimum cost-share, i.e., $\xi\left(i, S_{i}\right)=\xi^{*}(i, L, U)$. Note since $\xi(i, S)>\xi^{*}(i, L, U)=\xi\left(i, S_{i}\right)$, it must be that $\xi(i, S)=1$ and $\xi\left(i, S_{i}\right)=1 / 2$. Thus, by the definiton of our cost-sharing scheme, $i$ is matched in maximum matching $M_{S_{i}}$ (i.e., $i \in V\left(S_{i}\right)$ ) and unmatched in maximum matching $M_{S}$ (i.e., $\left.i \notin V(S)\right)$. Consider the alternating path $\left(j_{1}, \ldots, j_{m}\right)$ of maximum length starting from vertex $j_{1}=i$ whose odd edges belong to $M_{S_{i}}$ and whose even edges belong to $M_{S}$. We show that we can find an agent $j$ as required by the lemma which we remove or add depending on its parity in the path. 
Case 1: Suppose $m$ is odd. Note since $i \in V\left(S_{i}\right)$, this implies $m \geq 3$. Thus the path ends in an even edge $\left(j_{m-1}, j_{m}\right)$ where $j_{m} \in V(S)$ and $j_{m} \notin V\left(S_{i}\right)$. We would to show that we can delete $j_{m}$ and deduce the first inequality, but in order to do so we must first argue $j_{m} \notin L$. To do so, we show that $j_{m} \notin S_{i}$ and consequently $j_{m} \notin L$. Assume for contradiction that $j_{m} \in S_{i}$. Notice that by the definition of the alternating path $j_{1}, \ldots, j_{m-1} \in S_{i}$ while $j_{2}, \ldots, j_{m} \in S$. Since the first vertex $j_{1}=i \in L$ it follows that $j_{1} \in S$ as well. Moreover, we already assumed that $j_{m} \in S_{i}$. As a result, every vertex in this path belongs to $S \cap S_{i}$. Now one of the matchings projected to the vertices along this path must come lexicographically first. Assume that the projection corresponding to $M_{S}$ comes first (the other case is similar). Then since $j_{m}$ is not matched in $S_{i}$, we can construct a matching that comes lexicographically before $M_{S_{i}}$ and has equal cardinality by leaving $j_{1}=i$ unmatched, matching agents $j_{2}, \ldots, j_{m}$ according to $M_{S}$, and keeping the rest of the agents as they were matched in $M_{S_{i}}$, i.e.,

$$
\left(M_{S_{i}} \backslash\left\{\left(j_{1}, j_{2}\right), \ldots,\left(j_{m-2}, j_{m-1}\right)\right\}\right) \cup\left\{\left(j_{2}, j_{3}\right), \ldots,\left(j_{m-1}, j_{m}\right)\right\} .
$$

This contradicts the fact that $M\left(S_{i}\right)$ was the lexicographically first maximum matching in the graph induced by agents in $S_{i}$. Thus $j_{m} \notin S_{i}$ and so $j_{m} \notin L$. Note by the above argument, $L \subseteq S \backslash\left\{j_{m}\right\} \subseteq U$. Since $j_{1} \in L$, it follows that $j_{1} \in S$ and $j_{1} \in S \backslash\left\{j_{m}\right\}$, and so by removing the matched element $j_{m}$, we can construct a matching for $S \backslash\left\{j_{m}\right\}$,

$$
\left(M_{S} \backslash\left\{\left(j_{2}, j_{3}\right), \ldots,\left(j_{m-1}, j_{m}\right)\right\}\right) \cup\left\{\left(j_{1}, j_{2}\right), \ldots,\left(j_{m-2}, j_{m-1}\right)\right\},
$$

that has the same cardinality as $M_{S}$.

Case 2: Suppose $m$ is even, i.e., $j_{m} \in V\left(S_{i}\right)$ and $j_{m} \notin V(S)$. This time we add $j_{m}$ to $S$ and deduce the second inequality, but to do so must first argue that $j_{m} \notin S$. This is the case since if $j_{m} \in S$ then this alternating path is also an augmenting path, which contradicts the fact that $M_{S}$ has maximum cardinality. Now consider $S \cup\left\{j_{m}\right\}$ and note that the set of edges

$$
\left(M_{S} \backslash\left\{\left(j_{2}, j_{3}\right), \ldots,\left(j_{m-2}, j_{m-1}\right)\right\}\right) \cup\left\{\left(j_{1}, j_{2}\right), \ldots,\left(j_{m-1}, j_{m}\right)\right\}
$$

is a valid matching for $S \cup\left\{j_{m}\right\}$ and has one greater cardinality than $M_{S}$.

We are now ready to prove our main theorem which shows that the cost-sharing scheme satisfies the properties for yielding a group-strategyproof mechanism.

Proof of Theorem 3. We show the scheme satisfies the three properties of Theorem 1. Consider arbitrary sets $L$ and $U$ with $L \subseteq U$.

Property (a): We first show that we can find a set $S^{*}$ with $L \subseteq S^{*} \subseteq U$ such that for all $i \in S^{*}, \xi\left(i, S^{*}\right)=\xi^{*}(i, L, U)$. We start with an arbitrary set $S$ with $L \subseteq S \subseteq U$. Assume, wlog, for all $i \in S \backslash L, \xi(i, S)=1 / 2$ (if not, remove all such agents with $\xi(i, S)=1$; this does not change the others' cost-shares by Corollary 1$)$. Since the only cost-shares charged to the agents are $1 / 2$ and 1 , this implies that $\xi(i, S)=\xi^{*}(i, L, U)=1 / 2$ for all $i \in S \backslash L$. Now suppose there is some $i \in S \cap L$ with $\xi(i, S)>\xi^{*}(i, L, U)$ (if not, we have found a set $S^{*}$ satisfying the conditions of property (a)). By Lemma 2 there is either a matched element $j \in S \backslash L$ such that $|V(S)| \leq|V(S \backslash\{j\})|$ in which case the number of unmatched vertices (i.e., those with cost-share equal to 1) strictly decreases:

$$
|\{k \in S \mid \xi(k, S)=1\}|>|\{k \in S \mid \xi(k, S \backslash\{j\})=1\}|
$$


(the inequality is strict since $j$ is matched), or there is an element $j \in U \backslash S$ such that $|V(S)|<$ $|V(S \cup\{j\})|$ in which case, similarly,

$$
|\{k \in S \mid \xi(k, S)=1\}|>|\{k \in S \mid \xi(k, S \cup\{j\})=1\}| .
$$

Since we assumed that only players in $L$ are charged more than $1 / 2$, in either case this implies that the quantity $|\{k \in L \mid \xi(k, S)=1\}|$ strictly decreases. Since the number of vertices in $L$ is fixed and finite, this process must terminate and produce a set $S^{*}, L \subseteq S^{*} \subseteq U$, such that for all $i \in S^{*}$, $\xi\left(i, S^{*}\right)=\xi^{*}(i, L, U)$.

Property (b): Next we show for every player $i \in U \backslash L$, there is a set $S_{i}$ such that all agents $j \in S_{i} \backslash L$ have $\xi\left(j, S_{i}\right)=\xi^{*}(j, L, U)$. Consider some $i \in U \backslash L$. In the case where $\xi^{*}(i, L, U)=1$ then the set $S_{i}=L \cup\{i\}$ satisfies the property trivially, as player $i$ cannot be charged a greater amount in any set. Assume that $\xi^{*}(i, L, U)=1 / 2$ and let $S$ be a set where $L \subseteq S \subseteq U, i \in S$, and $\xi(i, S)=1 / 2$. Let $T=\{j \in S \backslash L \mid \xi(j, S)=1\}$ and set $S_{i}=S \backslash T$. By Corollary 1, all agents $k \in S_{i} \cap S$ have $\xi\left(k, S_{i}\right)=\xi(k, S)=1 / 2$. Thus for all $k \in S_{i} \backslash L, \xi\left(k, S_{i}\right)=\xi^{*}(k, L, U)=1 / 2$, as this is the lowest amount they may be charged by the cost-sharing scheme.

Property (c): Finally, we show that if for some $C \subset U$, there is a player $j \in C$ with $\xi(j, C)<$ $\xi^{*}(j, L, U)$, then there is a set $T \neq \emptyset$ with $T \subseteq L \backslash C$ such that for all $i \in T, \xi(i, C \cup T)=\xi^{*}(i, L, U)$. Consider some $C \subset U$ and some $j \in C$ such that $\xi(j, C)<\xi^{*}(j, L, U)$. Notice that this is only possible if $\xi(j, C)=1 / 2$ and $\xi^{*}(j, L, U)=1$. If for all agents $i \in L \backslash C, \xi^{*}(i, L, U)=1$, then the set $T=L \backslash C$ trivially meets the requirements. Otherwise we look for agents $i \in T \subseteq L \backslash C$ with $\xi(i, T \cup C)=\xi^{*}(i, L, U)=1 / 2$. To find $T$, we look at the set $L \cup C$ and discard players in $L \backslash C$ who have cost-share 1: namely, let $Q=\{i \in L \backslash C \mid \xi(i, L \cup C)=1\}$ and let $T=L \backslash(C \cup Q)$. First note by Corollary 1, for all $i \in T, \xi(i, T \cup C)=\xi(i, L \cup C)=1 / 2$. Hence for all $i \in T$, $\xi(i, T \cup C)=\xi^{*}(i, L, U)$. To show that this meets the requirement of property (c), it remains to show that $T$ is non-empty. Namely, we claim that there is some $i \in L \backslash C$ such that $\xi(i, L \cup C)=1 / 2$. Otherwise, $V\left(M_{L \cup C}\right) \subseteq C$, i.e., only players in $C$ are contained in the maximum matching which implies that $M_{C}=M_{L \cup C}$ and consequently $V\left(M_{C}\right)=V\left(M_{L \cup C}\right)$. Since $j \in V\left(M_{C}\right)$ this implies that $\xi(j, L \cup C)=1 / 2$, contradicting our observation that $\xi^{*}(j, L, U)=1$.

\subsection{Polynomial-Time Allocation}

We now argue that the group-strategyproof mechanism corresponding to the cost-sharing scheme in Definition 3 can be constructed in polynomial time. To do so, we must find, for any bid vector $b$, a set $S$ satisfying the conditions of Theorem 2. Namely, we are looking for a set $S$ that lies between some lower-bound set $L(b)$ and upper-bound set $U(b)$ such that:

1. for all $i \in L(b), b_{i}>\xi^{*}(i, L(b), U(b))$,

2. for all $i \in U(b) \backslash L(b), b_{i}=\xi^{*}(i, L(b), U(b))$,

3. and for all $R \subseteq \mathcal{A} \backslash U(b)$, there exist $i \in R$ with $b_{i}<\xi^{*}(i, L(b), U(b) \cup R)$,

and for all $i \in S, \xi(i, S)=\xi^{*}(i, L(b), U(b))$. In words, the elements in $L(b)$ should be bidding more than their minimum cost-share; the elements in $U(b) \backslash L(b)$ should be bidding equal to their minimum cost-share; and $U(b)$ is maximal in the sense that when we try to add elements to it, at least one of the newcomers can't afford his minimum cost-share. The set $S$ allocated by the group-strategyproof mechanism is then any of the intermediate sets in which each agent is happy (gets its minimum cost-share), i.e., a set $S$ with

$$
L(b) \subseteq S \subseteq U(b), \text { s.t. } \forall i \in S, \xi(i, S)=\xi^{*}(i, L(b), U(b)) .
$$


For ease of notation, in the rest of this section we fix $b$ and use $L$ to denote $L(b)$ and $U$ to denote $U(b)$.

The main difficulty in finding $S$ is that we do not know $L$ and $U$. However, using the structure of these sets and the fact that the only cost-shares in our scheme are 1 and $1 / 2$, we can bound these two sets. Given a bid vector $b$ let us define $P=\left\{i \mid b_{i}>\frac{1}{2}\right\}$ and $Q=\left\{i \mid b_{i} \geq \frac{1}{2}\right\}$. Then $L \subseteq P$ and $U \subseteq Q$. Hence we can search through intermediate sets of $P$ and $Q$, looking for our $S$. Any such $S$ will definitely contain $L$ as $L \subseteq P$, but may not be contained in $U$; our algorithm must provide a separate guarantee for this containment.

Our algorithm for finding $S$ is based on a local search procedure and corresponding potential function $\phi($.$) which is strictly increasing with respect to the steps of this search. The search$ procedure iteratively adds an element to, or deletes an element from, the current set $S$ while maintaining the invariant that $P \subseteq S \subseteq Q$. Our potential function $\phi(S)$ counts the number of happy elements in $L \subseteq S$, i.e.,

$$
\phi(S)=\left|\left\{i \in L \mid \xi(i, S)=\xi^{*}(i, L, U)\right\}\right| .
$$

Using our key lemma, Lemma 2, we show that as long as $\phi(S)<|L|$, there is always a way to improve the potential. Since $L$ is fixed and finite (given $b$ ), this procedure must terminate. Furthermore, by definition of the potential, when the procedure terminates, each agent in $L \subseteq S$ is happy. To guarantee that agents in $S \backslash L$ are happy and also that $S \subseteq U$, we need to prune $S$. As we show later, it is sufficient to simply remove agents from $S$ whose bids are less than their cost-shares. The following procedure implements this local search.

1. $S \leftarrow P$.

2. Iterate as long as the set $S$ changes:

(a) Remove all players in $S \backslash P$ with $\xi(i, S)=1$.

(b) If there is some $i \in Q \backslash S$ such that the cardinality of the maximum matching in $S \cup\{i\}$ is increased, then set $S \leftarrow S \cup\{i\}$.

(c) Else if there is some $i \in S \backslash P$ that was matched in $M(S)$ and the maximum matching in $S \backslash\{i\}$ does not decrease, then set $S \leftarrow S \backslash\{i\}$.

3. Set $S \leftarrow\left\{i \mid b_{i} \geq \xi(i, S)\right\}$.

We first observe that this algorithm runs in polynomial time. Specifically steps 2 (b) and 2 (c) reduce to finding whether the inclusion of some agent in $Q \backslash P$ forms an augmented path or wether an agent in $S \backslash P$ is not present in every maximum cardinality matching respectively. Both of these steps can be implemented in polynomial time. Since at each step the potential function increases, step 2 is performed at most as many times as the cardinality of $L$, which is bounded by the total number of agents.

We next prove that when the algorithm terminates, the set $S$ satisfies the conditions of Theorem 2. The following two lemmas are used in the proof. The first is used to prove that the pruning in step 3 forces $S$ to be contained in $U$. The second is used to argue that the potential function is increasing.

Lemma 3. If for some $S, L \subseteq S$, and for all $i \in S \backslash L, \xi(i, S) \leq b_{i}$, then $S \subseteq U$.

Proof. Consider some such $S$ and any $i \in S \backslash L$. Note $\xi^{*}(i, L, U \cup S) \leq \xi(i, S) \leq b_{i}$. Thus by the third condition for $L$ and $U$, we must have that $S \subseteq U$. 
Lemma 4. For any $S, P \subseteq S \subseteq Q$, if there is an agent $i \in P$ that is matched in $M_{S}$, then $i \in L$ and $\xi(i, S)=\xi^{*}(i, L, U)$.

Proof. Let $S^{\prime}=L \cup\left\{j \in S \mid \xi(j, S)=\frac{1}{2}\right\}$, i.e., we removed all the agents in $S \backslash L$ that are charged 1. By Corollary 1 for all $j \in S^{\prime}, \xi\left(j, S^{\prime}\right)=\xi(j, S)$. Every agent in $S^{\prime} \backslash L$ is charged $\frac{1}{2}$ and also since $S^{\prime} \subseteq S \subseteq Q$ for every $i \in S^{\prime} \backslash L$ it holds that $b_{i} \geq \frac{1}{2}=\xi\left(i, S^{\prime}\right)$. Lemma 3 implies that $S^{\prime} \subseteq U$, hence, $i \in U$ and $\xi\left(i, S^{\prime}\right) \geq \xi^{*}(i, L, U) \Rightarrow \xi^{*}(i, L, U)=\frac{1}{2}$. Finally, our assumption that $L, U$ satisfy the conditions of Theorem 2 imply that $i \in L$.

Theorem 4. This procedure outputs a set $S, L \subseteq S \subseteq U$, where for all $i \in S, \xi(i, S)=\xi^{*}(i, L, U)$.

Proof. We first show that every time step 2 (b) or 2 (c) is performed, the potential function increases. After step 2 (a), every agent $i \in S \backslash P$ is paying $1 / 2$. Thus all $i \in S \backslash P$ are matched by $M_{S}$. Since by Lemma 2 the number of matched agents in the new set strictly increases, it follows that the number of agents in $P$ that are matched increases. By Lemma 4, this implies that the number of agents in $L$ that are charged $\xi^{*}(i, L, U)$ increases.

Next, we show that either step 2 (b) or step 2 (c) can always be performed as long as $\phi(S)<|L|$. First note that, by Lemma 4, if for some $i \in L \subseteq P, \xi(i, S)=1 / 2$, then $\xi(i, S)=\xi^{*}(i, L, U)$. Since all cost-shares are 1 or $1 / 2$, this means that $\xi(i, S) \geq \xi^{*}(i, L, U)$ for all $i \in L$ (even though $S$ may not lie between $L$ and $U)$. So if $\phi(S)<|L|$, there must be some $i \in L$ such that $\xi(i, S)>\xi^{*}(i, L, U)$. We apply Lemma 2 for $i$ at $L, Q$. Note that the Lemma requires that $\xi(i, S)>\xi^{*}(i, L, Q)$ whereas what we know is that $\xi(i, S)>\xi^{*}(i, L, U)$. However, since $U \subseteq Q$ the second statement implies the first.

Our previous arguments imply that for the final set $S, L \subseteq S$ and for all $i \in L, \xi(i, S)=$ $\xi^{*}(i, L, U)$. We still need to show that $S \subseteq U$ and for all $i \in S \backslash L, \xi(i, S)=\xi^{*}(i, L, U)$. Note that for all $i \in S, b_{i} \geq \xi(i, S)$. Hence, we can apply Lemma 3 to show that $S \subseteq U$. Moreover, for all $i \in S \backslash L, i \in U$ and so by the conditions of Theorem $2, \xi^{*}(i, L, U)=b_{i}=\xi(i, S)$ (where the latter equality follows as we've shown $L \subseteq S \subseteq U$ ).

\section{Set Cover}

In this section we show that it is impossible to construct a fully budget balanced group-strategyproof mechanism when the cost function is determined by the optimal objective function of the set-cover problem. Immorlica et al. [5] showed that no cross-monotonic cost-sharing scheme can have a budget-balance of better than $n^{-1 / 2}$ where $n$ is the number of elements or the size of the largest subset in the set-cover instance. Here we show that there are instances where no group-strategyproof mechanism can be (18/19)-budget-balanced. Thus, while group-strategyproof mechanisms may be able to improve upon the budget-balance factor of cross-monotonic ones, we show that they can not, in general, provide full budget-balance.

Definition 4. In the set cover game we are given a ground set $V$ and a collection of subsets $\mathcal{F} \subseteq 2^{V}$. The agents in the game are the elements $V$ of the ground set. Given a subset of agents $S \subseteq V$, a set-cover of $S$ is a collection of subsets $\mathcal{C} \subseteq \mathcal{F}$ such that $S \subseteq \bigcup_{C \in \mathcal{C}}$, i.e., every element $i \in S$ belongs to some subset $C \in \mathcal{C}$. The cost of a set of agents $S$ is the minimum cardinality set-cover of $S$. 
In the following subsections, in order to build intuition, we first prove that there is no fully budget-balanced group-strategyproof mechanism for the set-cover game. We then extend the proof to show the constant bound. Our counter-example uses the following instance of a setcover game. There are six elements $U=\left\{A_{1}, A_{2}, B_{1}, B_{2}, C_{1}, C_{2}\right\}$, and the collection of subsets is $\mathcal{F}=\left\{\left\{A_{i}, B_{j}, C_{k}\right\}_{i, j, k=1,2}\right\}$. In other words there are three groups of two elements and the available subsets are all those who contain exactly one element from each group.

\subsection{Impossibility of Full Budget-Balance}

We will show by contradiction that there is no fully budget balanced group-strategyproof mechanism for this instance of the set-cover game. The first step to reach a contradiction is to show that the necessary properties together with full budget balance imply that at sets of the form $\left\{A_{1}, A_{2}, B_{j}, C_{k}\right\}$, there must be an unfair sharing of the cost in the sense that $A_{1}$ or $A_{2}$ must be responsible for their externality (their inclusion increases the cost of the optimal solution by one). Since one of the agents of the group $A$ is responsible for her externality this puts an upper bound on the sum of the rest agents' payments. Then, since adding the missing agent from group $B$ does not increase the cost, we show this does not change the upper bound. Finally, we exploit the symmetric form of this instance to derive the same bound with different agents in groups $A$ and $B$. By summing, we bound the total contribution and yield the contradiction.

Our proofs rely heavily on the notions of good, bad, and neutral elements defined in Section 2.1. Recall that an element $i$ is good (bad, neutral, respectively) for element $j$ at set $S$ if removing $i$ from $S$ increases (decreases, keeps the same, respectively) the cost-share of $j$. In Proposition 1 , we derived some useful properties of the good, bad, and neutral relation. We start with some further observations.

By Proposition 1 (a), if $i$ is a bad element at $S$, then the cost share of every agent $j \in S \backslash\{i\}$ can only increase. Hence, the sum of the payments can only increase as well.

Corollary 2. If $i$ is bad for some $j \in S$, then $\sum_{j \in S \backslash\{i\}} \xi(j, S \backslash\{i\}) \geq \sum_{j \in S \backslash\{i\}} \xi(i, S)$.

An implication of this is the following.

Corollary 3. Let $\xi$ be a cost-sharing scheme that satisfies the conditions of Theorem 1, and suppose $\xi$ is fully budget balanced for a cost function $c$. Then if $c(S)=c(S \backslash\{i\})$, agent $i$ cannot be bad for every element $j \in S$ at $S$.

We first show either $A_{1}$ or $A_{2}$ pays a significant fraction of the cost of set $\left\{A_{1}, A_{2}, B_{j}, C_{k}\right\}$. The idea of the proof is to show that if neither agent carries the cost, then they are bad for other agents. This imposes bounds on various subsets and lets us argue that one of these subsets is not budget balanced.

Claim 1. For $j, k \in\{1,2\}$ and $S=\left\{A_{1}, A_{2}, B_{j}, C_{k}\right\}$, either $\xi\left(A_{1}, S\right) \geq 1$ or $\xi\left(A_{2}, S\right) \geq 1$.

Proof. Assume that the converse holds. Since $\xi\left(A_{1}, S\right)<1$, by budget balance we have that

$$
\xi\left(A_{2}, S\right)+\xi\left(B_{j}, S\right)+\xi\left(C_{k}, S\right)>1 .
$$

Hence, $A_{1}$ must be a bad element for at least one of the rest agents at $S=\left\{A_{1}, A_{2}, B_{j}, C_{k}\right\}$. By symmetry, we conclude tht $A_{2}$ must be bad for at least one other agent at $S$. We consider two cases: either $A_{1}$ and $A_{2}$ are both bad for the same element, or they are bad for different elements. 
Case 1: Suppose that $A_{1}$ and $A_{2}$ are both bad for the same element, say $B_{j}$, at $S$. Proposition 1 (b) implies that $\xi\left(A_{1},\left\{A_{1}, A_{2}, C_{k}\right\}\right)=\xi\left(A_{1},\left\{A_{1}, A_{2}, B_{j}, C_{k}\right\}\right)<1$ and $\xi\left(A_{2},\left\{A_{1}, A_{2}, C_{k}\right\}\right)=$ $\xi\left(A_{2},\left\{A_{1}, A_{2}, B_{j}, C_{k}\right\}\right)<1$. Using budget balance we conclude that $A_{1}$ is bad for at least one agent in $\left\{A_{2}, C_{k}\right\}$ and similarly $A_{2}$ is bad for at least one agent in $\left\{A_{1}, C_{k}\right\}$.

By Proposition 1 (b) it is impossible that both $A_{1}$ and $A_{2}$ are bad for each other. Hence, one of them, wlog $A_{1}$, must be bad for $C_{k}$. If $A_{2}$ is bad for $A_{1}$ then by Proposition 1 (c) she must be bad for $C_{k}$ as well. As a result both $A_{1}$ and $A_{2}$ must be bad for $C_{k}$.

Applying Proposition 1 (c) again we get that

$$
\xi\left(A_{1},\left\{A_{1}, A_{2}\right\}\right)=\xi\left(A_{1},\left\{A_{1}, A_{2}, C_{k}\right\}\right)<1
$$

and

$$
\xi\left(A_{2},\left\{A_{1}, A_{2}\right\}\right)=\xi\left(A_{2},\left\{A_{1}, A_{2}, C_{k}\right\}\right)<1,
$$

which contradicts budget balance.

Case 2: Suppose $A_{1}$ and $A_{2}$ are not both bad for the same element at $S=\left\{A_{1}, A_{2}, B_{j}, C_{k}\right\}$. Proposition 1 (b) excludes the case where $A_{1}$ and $A_{2}$ are bad for each other at $S$. Moreover, if one of them, say $A_{1}$ is bad for $A_{2}$, then by Proposition 1 (c) $A_{1}$ is also bad for whatever $A_{2}$ is bad for; hence, we are in the previous case. As a result, we can assume, wlog, that $A_{1}$ is bad for $B_{j}$ and neutral for $C_{k}$ at $\left\{A_{1}, A_{2}, B_{j}, C_{k}\right\}$, and $A_{2}$ is bad for $C_{k}$ and neutral for $B_{j}$ at $\left\{A_{1}, A_{2}, B_{j}, C_{k}\right\}$ (by Proposition 1 (a), neither can be good for any element).

Let $L=\left\{B_{j}, C_{k}\right\}$ and $U=\left\{A_{1}, A_{2}, B_{j}, C_{k}\right\}$. Property (a) of the characterization requires that there is a set $T$ with $L \subseteq T \subseteq U$ where every agent in $T$ is charged the minimum payment with respect to this restriction. By assumption, $T$ cannot be the set $S=\left\{A_{1}, A_{2}, B_{j}, C_{k}\right\}$, as $A_{1}$ and $A_{2}$ are bad for $B_{j}$ and $C_{k}$ respectively. Moreover, since $A_{1}$ is bad for $B_{j}$ and $A_{2}$ is neutral for $B_{j}$ at $\left\{A_{1}, A_{2}, B_{j}, C_{k}\right\}$ it holds that $\xi\left(B_{j},\left\{A_{1}, B_{j}, C_{k}\right\}\right)=\xi\left(B_{j},\left\{A_{1}, A_{2}, B_{j}, C_{k}\right\}\right)>\xi\left(B_{j},\left\{A_{2}, B_{j}, C_{k}\right\}\right)$. Thus we can exclude the possibility that the set $T$ equals $\left\{A_{1}, B_{j}, C_{k}\right\}$. Due to symmetry we also exclude the set $\left\{A_{2}, B_{j}, C_{k}\right\}$. Hence, the only set left is $\left\{B_{j}, C_{k}\right\}$. This implies that

$$
\xi\left(B_{j},\left\{A_{1}, B_{j}, C_{k}\right\}\right)+\xi\left(C_{k},\left\{A_{1}, B_{j}, C_{k}\right\}\right) \geq \xi\left(B_{j},\left\{B_{j}, C_{k}\right\}\right)+\xi\left(C_{k},\left\{B_{j}, C_{k}\right\}\right)
$$

As we argued before $C_{k}$ is charged an amount strictly greater than her minimum at $\left\{A_{1}, B_{j}, C_{k}\right\}$; hence, the previous inequality is strict. However, this is impossible since budget balance requires that $\xi\left(B_{j},\left\{B_{j}, C_{k}\right\}\right)+\xi\left(C_{k},\left\{B_{j}, C_{k}\right\}\right)=1$, and $\xi\left(B_{j},\left\{A_{1}, B_{j}, C_{k}\right\}\right)+\xi\left(C_{k},\left\{A_{1}, B_{j}, C_{k}\right\}\right) \leq 1$.

We next use this to make a claim about the payments of agents in sets with one added element (slowly working our way towards a bound on the payments of the agents in the entire set). The main idea is that (by Corollary 3) adding elements with zero marginal cost can not increase cost-shares.

Claim 2. Fix $k=1,2$ and let $S=\left\{A_{1}, A_{2}, B_{1}, B_{2}, C_{k}\right\}$. Then it holds that

$$
\sum_{x \in S} \xi(x, S)+\xi\left(C_{k}, S\right) \leq 2 .
$$

Proof. Consider set $\left\{A_{1}, A_{2}, B_{1}, C_{k}\right\}$. By Claim 1, at least one $A$-type agent has cost-share at least 1 , say $A_{2}$, i.e., $\xi\left(A_{2},\left\{A_{1}, A_{2}, B_{1}, C_{k}\right\}\right) \geq 1$. In this case, we say $A_{2}$ carries the cost of set $\left\{A_{1}, A_{2}, B_{1}, C_{k}\right\}$ (i.e., in this set, $A_{2}$ carries the cost of the externality). Then, budget balance implies that

$$
\sum_{x \in\left\{A_{1}, B_{1}, C_{k}\right\}} \xi\left(x,\left\{A_{1}, A_{2}, B_{1}, C_{k}\right\}\right) \leq 1
$$


Since $c\left(\left\{A_{1}, A_{2}, B_{1}, B_{2}, C_{k}\right\}\right)=c\left(\left\{A_{1}, A_{2}, B_{1}, C_{k}\right\}\right)$, Corollary 3 implies that agent $B_{2}$ cannot be bad for any element at $\left\{A_{1}, A_{2}, B_{1}, B_{2}, C_{k}\right\}$. Therefore, we conclude that if $A_{2}$ carries the cost of $\left\{A_{1}, A_{2}, B_{1}, C_{k}\right\}$, then the agents $\left\{A_{1}, B_{1}, C_{k}\right\}$ collectively don't pay too much in $\left\{A_{1}, A_{2}, B_{1}, B_{2}, C_{k}\right\}$ :

$$
\sum_{x \in\left\{A_{1}, B_{1}, C_{k}\right\}} \xi\left(x,\left\{A_{1}, A_{2}, B_{1}, B_{2}, C_{k}\right\}\right) \leq 1 .
$$

By symmetry a similar equation holds if we start with any of the four sets $\left\{A_{1}, A_{2}, B_{1}, C_{k}\right\}$, $\left\{A_{1}, A_{2}, B_{2}, C_{k}\right\},\left\{A_{1}, B_{1}, B_{2}, C_{k}\right\}$, and $\left\{A_{2}, B_{1}, B_{2}, C_{k}\right\}$. Furthermore, by Claim 1 , the cost of each set must be carried by some element: the first by some $A_{i}$, the second by some (possibly different) $A_{i^{\prime}}$, the third by some $B_{j}$, and the fourth by some (possibly different) $B_{j^{\prime}}$.

We claim that two of these induced equations are complementary in the sense their sum yields the claim. If the costs of sets $\left\{A_{1}, A_{2}, B_{1}, C_{k}\right\}$ and $\left\{A_{1}, A_{2}, B_{2}, C_{k}\right\}$ are carried by different $A_{i}$ then they are complementary. Otherwise, if both are carried by $A_{1}$, the equation induced by $\left\{A_{2}, B_{1}, B_{2}, C_{k}\right\}$ is complementary with one of them (no matter which $B_{j}$ carries the cost of $\left.\left\{A_{2}, B_{1}, B_{2}, C_{k}\right\}\right)$. On the other hand, by symmetry, if both are carried by $A_{2}$ then the equation induced by $\left\{A_{1}, B_{1}, B_{2}, C_{k}\right\}$ is complementary with one of them. Adding these complementary equations we derive the equation in the statement of the claim.

Our main result follows directly from the above claim.

Theorem 5. There is no fully budget-balanced group-strategyproof mechanism for the set-cover game.

Proof. Budget balance requires that

$$
\sum_{x \in\left\{A_{1}, A_{2}, B_{1}, B_{2}\right\}} \xi\left(x,\left\{A_{1}, A_{2}, B_{1}, B_{2}, C_{k}\right\}\right)=2
$$

This is only possible if the equation of Claim 2 holds with equality and $\xi\left(C_{k},\left\{A_{1}, A_{2}, B_{1}, B_{2}, C_{k}\right\}\right)=$ 0 . We can similarly apply Corollary 3 to show that $\xi\left(C_{k},\left\{A_{1}, A_{2}, B_{1}, B_{2}, C_{1}, C_{2}\right\}\right)=0$. By symmetry, we can conclude that the cost-share of every agent in $\left\{A_{1}, A_{2}, B_{1}, B_{2}, C_{1}, C_{2}\right\}$ is zero which contradicts budget balance.

\subsection{A constant lower bound}

The same example can be used to derive a constant bound on the budget balance factor of groupstrategyproof mechanisms for the set-cover game. The main complication in extending the proof is that Corollary 3 no longer holds. Instead we must use different arguments to bound payments.

Assume that there is a $\omega(1-\beta)$ budget-balanced mechanism. We show that $\beta>\frac{1}{19}$, which implies a bound of $\frac{18}{19}$ for the budget balance. Assume that the converse holds, i.e., $\beta \leq \frac{1}{19}$. The next claim generalizes Claim 1.

Claim 3. For $j, k \in\{1,2\}$ and $S=\left\{A_{1}, A_{2}, B_{j}, C_{k}\right\}$, either $\xi\left(A_{1}, S\right)>1-2 \beta$ or $\xi\left(A_{2}, S\right)>1-2 \beta$.

Proof. Given the budget balance assumption, a payment less or equal to $1-2 \beta$ for agent $A_{1}$ or $A_{2}$ at this set would guarantee that she is a bad element for one of the rest at $S$.

When $A_{1}, A_{2}$ is bad for $B_{j}$ or $C_{j}$ a similar argument can be used to show that $A_{1}$ and $A_{2}$ are both charged an amount less than or equal to $1-2 \beta$ after removing the element for which they 
are both bad, wlog $C_{j}$. Similarly, budget balance implies that they are bad for the other element $B_{j}$ at $\left\{A_{1}, A_{2}, B_{j}\right\}$, and we get

$$
\xi\left(A_{1},\left\{A_{1}, A_{2}\right\}\right)+\xi\left(A_{2},\left\{A_{1}, A_{2}\right\}\right)<2-4 \beta,
$$

which contradicts budget balance.

As in Claim 1, we only need to consider the case where $A_{1}$ is bad for $B_{j}$ but not bad for $C_{j}$ and $A_{2}$ is bad for $C_{j}$, and also $B_{j}$ and $C_{k}$ are not bad for each other. The same arguments imply that agents $B_{j}$ and $C_{k}$ achieve their minimum payment at $\left\{B_{j}, C_{k}\right\}$, and we get a similar equation

$$
\xi\left(B_{j},\left\{A_{1}, B_{j}, C_{k}\right\}\right)+\xi\left(C_{k},\left\{A_{1}, B_{j}, C_{k}\right\}\right)>\xi\left(B_{j},\left\{B_{j}, C_{k}\right\}\right)+\xi\left(C_{k},\left\{B_{j}, C_{k}\right\}\right)
$$

This equation does not imply a contradiction, as it did it the case of full budget balance since it is possible that $\xi\left(B_{j},\left\{A_{1}, B_{j}, C_{k}\right\}\right)+\xi\left(C_{k},\left\{A_{1}, B_{j}, C_{k}\right\}\right)>1-\beta$. This time we have to employ Property (b) of the characterization to reach a contradiction. Note that the previous equation implies that $\xi\left(A_{1},\left\{A_{1}, B_{j}, C_{k}\right\}\right)<\beta$ and by symmetry $\xi\left(A_{2},\left\{A_{2}, B_{j}, C_{k}\right\}\right)<\beta$.

Let $L=\emptyset$ and $U=\left\{A_{1}, A_{2}, B_{j}, C_{k}\right\}$. Our previous arguments imply the following bounds on the minimum payment of $A_{1}$ and $A_{2}: \xi^{*}\left(A_{1}, L, U\right)<\beta$ and $\xi^{*}\left(A_{2}, L, U\right)<\beta$. By Property (b) of the characterization, we have to find two sets $S_{A_{1}}$ and $S_{A_{2}}$ in this restriction such that $A_{1} \in S_{A_{1}}$, $A_{2} \in S_{A_{2}}$, and every agent in $S_{A_{1}} \backslash L$ and $S_{A_{2}} \backslash L$ (which equal $S_{A_{1}}$ and $S_{A_{2}}$ respectively since $L$ is empty) is charged her minimum payment with respect to this restriction.

Our assumptions exclude the sets $\left\{A_{1}, B_{j}, C_{k}\right\},\left\{A_{1}, B_{j}, C_{k}\right\},\left\{A_{1}, A_{2}, B_{j}\right\}$, and $\left\{A_{1}, A_{2}, C_{k}\right\}$ since either $B_{j}$ or $C_{k}$ are not charged their minimum payment. Moreover, we exclude the set $\left\{A_{1}, A_{2}\right\}$ since then the sum $\xi\left(A_{1},\left\{A_{2}, A_{2}\right\}\right)+\xi\left(A_{2},\left\{A_{1}, A_{2}\right\}\right)$ should be strictly less than $2 \beta$ and also strictly greater than $2-2 \beta$, which is impossible as we assumed that $\beta \leq \frac{1}{19}$.

The only sets left are those that contain one agent from group $A$ and either $B_{j}$ or $C_{k}$. We show that it is impossible that agents $A_{1}$ and $A_{2}$ achieve their minimum payment at set with a different agent. Note that the minimum payment of at least one $B_{j}$ or $C_{k}$ must be less than $\frac{1}{2}$, as the sum of their minimum values cannot be larger than 1 by budget balance. Wlog, assume that $\xi^{*}\left(B_{j}, L, U\right) \leq \frac{1}{2}$. If we could set $S_{A_{1}}=\left\{A_{1}, B_{j}\right\}$ it should be that the sum of the agents is less than $\frac{1}{2}+\beta$ but also larger than $1-\beta$, which is again impossible by our assumption that $\beta \leq \frac{1}{19}$.

As a result, $S_{A_{1}}=\left\{A_{1}, C_{k}\right\}$ and $S_{A_{2}}=\left\{A_{2}, C_{k}\right\}$, which implies agent $C_{k}$ is charged her minimum value at these two sets. We use this fact to show that one of the agents $A_{1}$ or $A_{2}$ is charged strictly less than $\beta$ at $\left\{A_{1}, A_{2}\right\}$. Then budget balance and our assumption about $\beta$ would imply that one of these agents in good while the other is bad contradicting Proposition 1 (b): Assume $\xi\left(A_{2},\left\{A_{1}, A_{2}\right\}\right)<\beta$ and by budget balance $\xi\left(A_{1},\left\{A_{1}, A_{2}\right\}\right)>2-3 \beta$. Since $\beta \leq \frac{1}{19}$ budget balance implies that $\xi\left(A_{2},\left\{A_{1}, A_{2}\right\}\right)<1-\beta<\xi\left(A_{2},\left\{A_{2}\right\}\right)$, hence, $A_{1}$ is good for $A_{2}$ at $\left\{A_{1}, A_{2}\right\}$. Moreover since $\beta \leq \frac{1}{19}$, budget balance implies that $\xi\left(A_{1},\left\{A_{1}, A_{2}\right\}\right)>1 \geq \xi\left(A_{1},\left\{A_{1}\right\}\right)$, i.e., $A_{2}$ is bad for $A_{1}$ at $\left\{A_{1}, A_{2}\right\}$.

We finish our proof by showing that our assumptions imply that either $A_{1}$ or $A_{2}$ are charged less than $\beta$ at $\left\{A_{1}, A_{2}\right\}$, thus, reaching a contradiction. We know that $C_{k}$ is charged an amount strictly greater than her minimum value at $\left\{A_{1}, A_{2}, C_{k}\right\}$, which implies that agents $A_{1}$ and $A_{2}$ are both bad for $C_{j}$. Proposition 1 (b) implies that $C_{j}$ is neutral for both of these agents, hence, $\xi\left(A_{1},\left\{A_{1}, A_{2}, C_{k}\right\}\right)<\beta$ and $\xi\left(A_{2},\left\{A_{1}, A_{2}, C_{k}\right\}\right)<\beta$. Moreover, Proposition 1 (a) implies that neither $A_{1}$ nor $A_{2}$ can be good for the other. Therefore, by Proposition 1 (b), one of them must be neutral, i.e., either $\xi\left(A_{1},\left\{A_{1}, A_{2}\right\}\right)=\xi\left(A_{1},\left\{A_{1}, A_{2}, C_{k}\right\}\right)<\beta$ or $\xi\left(A_{2},\left\{A_{1}, A_{2}\right\}\right)=$ $\xi\left(A_{2},\left\{A_{1}, A_{2}, C_{k}\right\}\right)<\beta$ which completes our proof. 
Using this claim we deduce a similar equation like in Claim 2. However, we must be more careful, as we cannot use Corollary 3 anymore.

Claim 4. Fix $k=1,2$ and let $S=\left\{A_{1}, A_{2}, B_{1}, B_{2}, C_{k}\right\}$. Then it holds that

$$
\sum_{x \in S} \xi(x, S)+\xi\left(C_{k}, S\right) \leq 2+4 \beta .
$$

Proof. Like before consider the set $\left\{A_{1}, A_{2}, B_{1}, C_{k}\right\}$. Similarly, let us assume that $A_{2}$ carries the cost of the externality, i.e., $\xi\left(A_{2},\left\{A_{1}, A_{2}, B_{1}, C_{k}\right\}\right) \geq 1-2 \beta$ which implies that

$$
\sum_{x \in\left\{A_{1}, A_{2}, B_{j}, C_{k}\right\}} \xi\left(x,\left\{A_{1}, A_{2}, B_{2}, C_{k}\right\}\right) \leq 1+2 \beta .
$$

If we show that

$$
\sum_{x \in\left\{A_{1}, A_{2}, B_{j}, C_{k}\right\}} \xi\left(x,\left\{A_{1}, A_{2}, B_{1}, B_{2}, C_{k}\right\}\right) \leq 1+2 \beta
$$

then we can use the same arguments regarding the existence of two complementary equations and derive the equation in the statement of the claim. If agent $B_{2}$ is not bad for any of the agents $A_{1}, B_{2}$, and $C_{k}$ then Equation 1 holds. If $B_{2}$ is bad for some of these agents then by Proposition 1 (a) she must be neutral or bad for $A_{2}$, i.e.,

$$
\xi\left(A_{2},\left\{A_{1}, A_{2}, B_{1}, B_{2}, C_{k}\right\} \geq \xi\left(A_{2},\left\{A_{1}, A_{2}, B_{j}, C_{k}\right\}>1-2 \beta .\right.\right.
$$

Hence we can derive Equation 1 by budget balance.

Subtracting the budget balance equation we get that

$$
\xi\left(C_{k},\left\{A_{1}, A_{2}, B_{1}, B_{2}, C_{k}\right\}\right) \leq 6 \beta .
$$

Again since we cannot use Corollary 3 we have to use different arguments to bound the payment of agent $C_{k}$ at the set $\left\{A_{1}, A_{2}, B_{1}, B_{2}, C_{1}, C_{2}\right\}$.

This time we bound the sum of the payments of agents $C_{1}$ and $C_{2}$ and particularly we show that

$$
\xi\left(C_{1},\left\{A_{1}, A_{2}, B_{1}, B_{2}, C_{1}, C_{2}\right\}\right)+\xi\left(C_{2},\left\{A_{1}, A_{2}, B_{1}, B_{2}, C_{1}, C_{2}\right\}\right) \leq 12 \beta .
$$

If $C_{2}$ is good for $C_{1}$ at $\left\{A_{1}, A_{2}, B_{1}, B_{2}, C_{1}, C_{2}\right\}$, then the contrapositive of Proposition 1 (b) implies that $C_{1}$ is good or neutral for $C_{2}$ and the result follows. Now if $C_{2}$ is bad for $C_{1}$ then by Corollary 2 the sum of the payments of agents in $\left\{A_{1}, A_{2}, B_{1}, B_{2}, C_{1}\right\}$ has to increase. Let $\Delta$ be the denote this difference, i.e.,

$$
\Delta=\sum_{x \in\left\{A_{1}, A_{2}, B_{1}, B_{2}, C_{1}\right\}} \xi\left(x,\left\{A_{1}, A_{2}, B_{1}, B_{2}, C_{1}, C_{2}\right\}\right)-\xi\left(x,\left\{A_{1}, A_{2}, B_{1}, B_{2}, C_{1}\right\}\right) .
$$

By Proposition 1 (a) the payment of every agent can only increase after the inclusion of agent $C_{2}$, hence, $\Delta$ is an upper bound on the increase in the payment of $C_{1}$. Moreover, note that by budget balance the payment of agent $C_{2}$ is less than 


$$
\begin{gathered}
2-\sum_{x \in\left\{A_{1}, A_{2}, B_{1}, B_{2}, C_{1}\right\}} \xi\left(x,\left\{A_{1}, A_{2}, B_{1}, B_{2}, C_{1}, C_{2}\right\}\right)= \\
2-\sum_{x \in\left\{A_{1}, A_{2}, B_{1}, B_{2}, C_{1}\right\}} \xi\left(x,\left\{A_{1}, A_{2}, B_{1}, B_{2}, C_{1}\right\}\right)-\Delta<
\end{gathered}
$$

$$
2 \beta-\Delta .
$$

As a result,

$$
\xi\left(C_{1},\left\{A_{1}, A_{2}, B_{1}, B_{2}, C_{1}, C_{2}\right\}\right)+\xi\left(C_{1},\left\{A_{1}, A_{2}, B_{1}, B_{2}, C_{1}, C_{2}\right\}\right) \leq 8 \beta
$$

which implies Equation 2.

Using the same arguments for $A_{1}, A_{2}$ and $B_{1}, B_{2}$ we get that

$$
\sum_{x \in\left\{A_{1}, A_{2}, B_{1}, B_{2}, C_{1}, C_{2}\right\}} \xi\left(x,\left\{A_{1}, A_{2}, B_{1}, B_{2}, C_{1}, C_{2}\right\}\right)<36 \beta .
$$

Budget blance implies that $36 \beta>2-2 \beta \Leftrightarrow \beta>\frac{1}{19}$, which contradicts our assumption.

\section{Conclusion}

Our work is the first application of the complete characterization of group-strategyproof mechanisms, given by Pountourakis and Vidali [13]. We use the characterization to show bounds on the budget balance of group-strategyproof mechanisms for specific combinatorial problems. While the case of edge-cover is completely solved by this paper, it remains open to bound the optimal budget balance factor of group-strategyproof mechanisms for set-cover. Other problems of interest include facility location, vertex cover, Steiner tree, and Steiner forest. In the previous literature, these problems have only been solved using techniques involving cross-monotonic cost-sharing schemes, and it is known such an approach can not achieve perfect budget-balance for these problems.

Many constructions of cross-monotonic cost-sharing schemes rely on primal-dual schema. In these schemes, the natural linear-programming formulations of the combinatorial problems have constraints corresponding to the demand of the agents. The primal-dual scheme charges each agent her respective dual variable. In order to guarantee cross-monotonicity, these schemes introduce the notion of ghost-shares, i.e., the idea that variables contributing to a tight constraint are not frozen but rather keep growing and contributing to other constraints. Nevertheless, the payment of an agent is determined by the time that the dual variable was first involved in a tight constraint.

If we don't use the ghost-share technique the resulting cost-sharing scheme is not cross-monotone. However, in many cases it can be used to design weakly group-strategyproof mechanisms [8]. A natural question that arises is whether such a cost-sharing scheme satisfies the necessary conditions of group-strategyproofness despite the fact that it fails to satisfy cross-monotonicity. Unfortunately, the following observation indicates that this may not be true. Consider a cost-sharing scheme that is constructed by a primal-dual scheme and does not satisfy cross-monotonicity. Note that this implies the existence of at least one agent $i$ that is bad for another agent $j$ at some set $S$. This means that the constraint that was responsible for freezing the dual variable of agent $j$ becomes tight at a later time when $i$ is present. This is only possible if there is another agent $k \in S$ that 
contributed to this constraint for subset $S \backslash\{i\}$; however, the inclusion of agent $i$ caused the variable of $k$ to freeze earlier, which means that the payment of $k$ has to decrease. As a result, agent $i$ is bad for $j$ and good for $k$ which violates Proposition 1 (a).

The previous observation indicates that one should search beyond primal-dual schemes in order to design group-strategyproof mechanisms that perform strictly better than mechanisms captured by the cross-monotonic framework. 


\section{References}

[1] Janina A. Brenner and Guido Schäfer. Cost sharing methods for makespan and completion time scheduling. In Wolfgang Thomas and Pascal Weil, editors, STACS, volume 4393 of LNCS, pages 670-681. Springer, 2007.

[2] David Buchfuhrer, Michael Schapira, and Yaron Singer. Computation and incentives in combinatorial public projects. In David C. Parkes, Chrysanthos Dellarocas, and Moshe Tennenholtz, editors, ACM Conference on Electronic Commerce, pages 33-42. ACM, 2010.

[3] Nikhil R. Devanur, Milena Mihail, and Vijay V. Vazirani. Strategyproof cost-sharing mechanisms for set cover and facility location games. Decision Support Systems, 39(1):11-22, 2005.

[4] Anupam Gupta, Jochen Könemann, Stefano Leonardi, R. Ravi, and Guido Schäfer. An efficient cost-sharing mechanism for the prize-collecting steiner forest problem. In Nikhil Bansal, Kirk Pruhs, and Clifford Stein, editors, SODA, pages 1153-1162. SIAM, 2007.

[5] Nicole Immorlica, Mohammad Mahdian, and Vahab S. Mirrokni. Limitations of crossmonotonic cost-sharing schemes. ACM Trans. Algorithms, 4(2):1-25, 2008.

[6] Kamal Jain and Vijay V. Vazirani. Applications of approximation algorithms to cooperative games. In STOC, pages 364-372, 2001.

[7] Jochen Könemann, Stefano Leonardi, Guido Schäfer, and Stefan H. M. van Zwam. A group-strategyproof cost sharing mechanism for the steiner forest game. SIAM J. Comput., 37(5):1319-1341, 2008.

[8] Aranyak Mehta, Tim Roughgarden, and Mukund Sundararajan. Beyond moulin mechanisms. Games and Economic Behavior, 67(1):125-155, September 2009.

[9] Herve Moulin. Incremental cost sharing: Characterization by coalition strategy-proofness. Social Choice and Welfare, (16):279-320, 1999.

[10] Herve Moulin and Scott Shenker. Strategyproof sharing of submodular costs: budget balance versus efficiency. Economic Theory, 18:511-533, 2001.

[11] Martin Pál and Éva Tardos. Group strategyproof mechanisms via primal-dual algorithms. In FOCS, pages 584-593. IEEE Computer Society, 2003.

[12] Christos H. Papadimitriou, Michael Schapira, and Yaron Singer. On the hardness of being truthful. In FOCS, pages 250-259. IEEE Computer Society, 2008.

[13] Emmanouil Pountourakis and Angelina Vidali. A complete characterization of groupstrategyproof mechanisms of cost-sharing. In Mark de Berg and Ulrich Meyer, editors, ESA (1), volume 6346 of Lecture Notes in Computer Science, pages 146-157. Springer, 2010.

[14] Michael Schapira and Yaron Singer. Inapproximability of combinatorial public projects. In Christos H. Papadimitriou and Shuzhong Zhang, editors, WINE, volume 5385 of Lecture Notes in Computer Science, pages 351-361. Springer, 2008. 